\title{
The time has come to incorporate a greater focus on rate of force development training in the sports injury rehabilitation process
}

\author{
Matthew Buckthorpe ${ }^{1,2,3}$ \\ Giulio Sergio Roi ${ }^{1,4}$ \\ 1 Isokinetic Medical Group, FIFA Medical Centre of \\ Excellence, Education \& Research Department, \\ Bologna, Italy \\ 2 Isokinetic Medical Group, FIFA Medical Centre of \\ Excellence, London, UK \\ 3 Southampton Football Club, Southampton, UK \\ ${ }^{4}$ Department of Neuroscience, Biomedicine and \\ Movement, University of Verona, Italy
}

Corresponding Author:

Matthew Buckthorpe

Isokinetic Medical Group

11 Harley Street

WG1 9PF London, United Kingdom

E-mail: M.Buckthorpe@isokinetic.com

\section{Summary}

This narrative and literature review discusses the relevance of Rate of Force Development (RFD) (the slope of the force time curve) for Return To Sport (RTS), its determinants and the influence of training practices on it expression, with the purpose to enhance clinicians' awareness of how RFD training may enhance RTS success. RFD is considered functionally more relevant than maximal muscle strength during certain very fast actions including rapid joint stabilisation following mechanical perturbation. Deficits in RFD are reported following conventional rehabilitation programmes despite full restoration of maximal strength, which may contribute to the less than satisfactory RTS outcomes reported in the literature. RFD determinants vary as a function of time from force onset with a diminishing role of maximal strength as the time available for force development decreases. Factors such as neural activation, fibre type composition and muscle contractile properties influence RFD also and to a much greater extent during the early periods of rapid force development. Conventional resistance training using moderate loads typical of most rehabilitation programmes is insufficient at restoring or enhancing RFD, thus incorporating periodised resistance training programmes and explosive training techniques in the final stages of rehabilitation prior to RTS is recommended. Level of evidence: $\mathrm{V}$.

KEY WORDS: explosive strength, injury prevention, maximal isometric voluntary strength, neural activation, rehabilitation.

\section{Introduction}

The muscles transform chemical energy into mechanical energy, so they are considered the motors of the neuromuscular system. The Central Nervous System is ultimately the controller of muscle activation, as each individual muscle acts as a slave performing relatively simple tasks in isolation as ordered. Collectively, the combination of muscle recruitment across multiple muscles can culminate in eloquent movements, athletic performances, and optimal protection from non-contact type injuries during challenging dynamic tasks. The ability of the neuromuscular system to produce static and dynamic actions is defined as strength ${ }^{1}$ and its expression depends upon many aspects including time available in the task at hand, the type of muscular contraction, the complexity of the movement, as well as the inertia that it is been applied to.

Developing the neuromuscular system to enhance the capabilities to produce force is one of the most important goals of athletic development programmes, injury prevention and rehabilitation. However the concept of strength is frequently miss-interpreted, and viewed as the ability to lift heavy objects, typically quantified as the peak force or torque measured during isometric or isokinetic testing ${ }^{2,3}$, or considering a number of lifts of a weight at a particular heavy load (repetition maximum testing), while in many athletic movement several dynamic actions are very quickly performed such as the stance phase in sprint running $(100 \mathrm{~ms})^{4}$, and the rapid re-stabilisation of joints following mechanical perturbation to prevent injury $(<50 \mathrm{~ms})^{5}$. These movements involve contraction times which are shorter than the time that it takes to produce the Maximal Isometric Voluntary Force (MIVF), that typically is in the order of $300 \mathrm{~ms}^{6}$. Hence, the capability to produce strength during rapid sporting tasks (i.e. explosive efforts) ${ }^{7}$ may be more dependent upon the ability to produce force rapidly, which can be measured as Rate of Force Development $(R F D)^{8}$, which is considered functionally more important than MIVF during certain rapid functional movements, including re-stabilizing the body following a loss of balance e-11. $^{\text {. }}$ 
From the above, it can be distinguished that there are two forms of strength production by a healthy or injured athlete: I) the maximal strength, that is the maximum amount of strength produced during isometric or slow dynamic movements with heavy loads, and II) the strength produced at the fastest rate characterising explosive strength of athletes, elderly individuals and patients, measured as RFD.

This narrative review discusses the role of RFD in athletic performance and injury prevention, together with its determinants and the influence of athletic training, with the overall purpose to enhance awareness of how the explosive strength capabilities may contribute to enhanced return to sport outcomes after injury with some guide as to how to implement RFD training into clinical practice. This paper complies to ethical and scientific standards according to the basic principles and recommendations of Muscle, Ligament and Tendon Journal7,12.

\section{Relevance of RFD in athletic performance}

As previously described, RFD is considered to be an important aspect of neuromuscular function during sports activities where time to develop force is limited (such as balance corrections, sprinting, jumping and punching $)^{9,10}$. Tillin, et al. ${ }^{10}$ have reported that power athletes with ability for very fast sporting actions (sprinters and jumpers) displayed two-fold superior ability to express their available explosive strength capability during the early phase of muscle contraction compared to untrained participants, but their MIVF was only $26 \%$ superior. Thereby, suggesting the ability for RFD is of more importance than that of differences in maximal strength for sporting events requiring rapid production of force.

The association of RFD and athletic performance depends on both the athletic tasks and the mode of assessment. There is a large body of equivocal findings concerning relationships between measured RFD and athletic sporting tasks ${ }^{13-15}$. It is now becoming clear that the time allowed for both the athletic task, and the time over which RFD is measured and interpreted are of importance in elucidating this shared variance. Tillin et al. ${ }^{11}$ reported RFD over distinct time periods from force onset $(50,100,150,200$ and $250 \mathrm{~ms})$ during rapid isometric squats and related force production at each time point to both countermovement jump and sprint performance. It was shown that RFD in the early phase of the squat ( $\leq 100 \mathrm{~ms}$ ) was more closely related to acceleration capabilities during sprint running $(5-20 \mathrm{~m}, \mathrm{r}=-0.54$ to -0.63$)$, whereas the ability for late phase squat RFD (>100 ms) was more related to vertical jump height ( $r=0.51$ to 0.61 ). This is likely due to the time available for force production during each task (sprint running 80-120 ms vs jumping, $250 \mathrm{~ms}$ ). Interestingly, when the athletes in the study were also split into two separate groups based on sprint times (one with $5 \mathrm{~m}$ sprint times $<1 \mathrm{~s}$ and the other group $>1 \mathrm{~s}$ ), there was no difference in squat MIVF between groups, whilst the faster group had superior relative RFD. The study demonstrates the importance of considering the time constraints in athletic sporting tasks when considering the neuromuscular contributions to performance.

\section{Relevance of RFD for return to sport and prevention of re-injury}

Joint stability is determined by the interaction between the dynamic joint stabilisers (musculature) and the passive joint structures (including ligaments) ${ }^{16}$. As an injury such as anterior cruciate ligament rupture occurs within $50 \mathrm{~ms}$ after ground contact ${ }^{5}$, it is likely that the ability to produce force rapidly ( 25-50 $\mathrm{ms}$ ) is of more importance than the MIVF capabilities to injury prevention. Thus, the role of early phase RFD as an injury risk factor may be of more clinical relevance than MIVF, but has yet to any receive any direct scientific attention.

There is accumulating indirect evidence that suggests that RFD could be an important factor in injury occurrence. RFD has been shown to be an important determinant of the effectiveness of postural corrections during gait and following a perturbation that could lead to a fall or injury ${ }^{17}$. Additionally, although by no means causative, balance training has been reported to both separately enhance RFD ${ }^{18}$ and decrease risk of joint injuries ${ }^{19}$ particularly in previously injured participants. Furthermore, fatigue has also been linked to an increased risk of injury ${ }^{20}$ and demonstrated to exert a more pronounced influence on RFD than MIVF 21 . Recently, it was reported six months following anterior cruciate ligament reconstruction that average MIVF had been restored to $97 \%$, whereas RFD at 30,50 and $90 \%$ MIVF were only 80,77 and $63 \%$, respectively of pre-injury 22 . Pre-injury RFD values were only achieved at 12 months after anterior cruciate ligament reconstruction, following a rehabilitation program focusing on muscle power. These Authors suggested that following an anterior cruciate ligament reconstruction, RFD criteria may be a useful adjunct outcome measure for the decision to return athletes to sports. Additionally, in a similar fashion, it was shown that those patients with previous history of hamstring muscle strain, reported $40 \%$ lower early phase (50$100 \mathrm{~ms}$ ) eccentric RFD of their previously injured versus their contralateral limb ${ }^{23}$.

Given that a high percentage of patients following anterior cruciate ligament reconstruction report less than satisfactory outcomes after rehabilitation with mediocre return to sport figures ${ }^{24}$, in addition to the high reported hamstring re-injury rates in the literature ${ }^{25}$, this deficit in RFD on return to sport could have important clinical implications in re-injury occurrence, and thus, be an important component for re-injury prevention. Theoretically, enhanced capability for RFD should exert a beneficial effect on injury resistance. 
The time has come to incorporate a greater focus on rate of force development training in the sports injury rehabilitation process

\section{Measurement of RFD for clinical practice}

RFD can be quantified as the force (or torque) produced at specific time points from contraction on$\operatorname{set}^{10}$, the RFD over a particular time period (i.e. the slope of the force time-curve $)^{7}$ the force-time integral (area beneath the force-time curve) ${ }^{9}$ or reported as the peak slope of the force-time curve, referred to as peak RFD. It is thought that reporting RFD over distinct periods $(0-50,50-100$ and $100-150 \mathrm{~ms})$ provides a more reliable measure and clearer understanding of the determinants of RFD ${ }^{11,26}$ or over distinct times windows, such as $50 \mathrm{~ms}^{10}$. RFD is typically measured isometrically using isometric dynamometer ${ }^{9}$, isometric strength testing rig $^{27}$ or on a force plate ${ }^{15,26,28}$. Some studies have attempted measurement of RFD during dynamic actions, however, this situation is experimentally problematic, as the mechanics of the system interact with velocity in a non-linear manner. Based on Newtonian physics (force $=$ mass $\times$ acceleration) and the force-velocity relationship an increase in force during the early phase of a dynamic action will result in an increase in acceleration and therefore a higher velocity, which will then comprise potential force production during latter time points of the dynamic action, thereby influencing the observed RFD throughout the dynamic contraction.

There are certain methodological factors which need to be considered when assessing RFD. These have been discussed in great detail previously ${ }^{8}$. A summary of the key points and implications for measurement of RFD in the rehabilitation process will be discussed here. It is apparent that system compliance negatively influences RFD but does so it reads RFD but does not necessarily influence MVF. Thus, a stiff measurement system is needed for accurate interpretation of RFD. Typically, isokinetic assessment of peak torque of the knee extensors and flexors is undertaken during the rehabilitation of knee injuries such as anterior cruciate ligament reconstruction. However, the typical configuration of commercially available isokinetic dynamometers with a large volume of padding is not suited for RFD measurement. As previously described, force and velocity are interrelated, and therefore velocity can have a strong impact on subsequent force production. Consequently, RFD is typically measured isometrically. The identification of force onsets is pivotal to the valid and reliable assessment of RFD. Most studies have determined force onset for explosive contractions using automated methods of onset such as absolute force thresholds or mathematical algorithms (e.g. 3 standard deviations of mean baseline force). However, manual (visual) identification of signal onsets is considered the "gold standard" method for identifying signal onsets. Therefore, automated detection methods are considerably less valid and relatively insensitive than manual (visual) identification procedures. Furthermore, the level of pre-tension in the muscle prior to performing an explosive contraction has been shown to subsequently affect the level of force that can be applied over the initial rising phase of contraction and so explosive contractions should be performed from a relaxed state (i.e. no pre-tension). Counter-movement (a drop-in baseline force) exerts a strong influence on subsequent recorded values of explosive force production and therefore a consistent baseline force should be ensured.

As such RFD measurement is not simple and numerous methodological factors can influence its interpretation. Additionally, reliability is a potential problem with RFD measurement. Buckthorpe et al. ${ }^{26}$ assessed RFD measurement and demonstrated that although MIVF was reliable for both individuals and group between sessions, early phase RFD was highly variable between sessions (coefficient of variation, 16-19\% depending upon the method of reporting), but consistent for the group. This reliability improved as time from force onset increased. As such caution is warranted when interpreting early phase RFD.

Whilst functional human movement involves the coordinated actions of multiple joints, given the number of technical considerations it can be seen why explosive force production has typically been assessed in isolated muscle groups ${ }^{9,29}$. A few studies have assessed RFD in multiple joint situations such as an isometric squat ${ }^{11}$ or leg press ${ }^{18}$. However, this situation is not ideal for understanding the underlying neural and morphological factors which may influence the expression of explosive strength between participants or between limbs following injury. Therefore, measurement of isometric explosive strength in a single joint isometric situation minimises the potential number of confounding variables that can influence RFD measurement, and allows for a more controlled situation in which the contributions to variance in RFD or morphological and neural contributions to changes in explosive strength following an intervention and/or injury can be more appropriately examined.

\section{Training RFD}

There are numerous barriers to the accurate measurement of RFD in clinical practice following injury prior to return to sport. As such for most clinicians this will not be possible. However, given the relevance of RFD for athletic performance and injury risk, consideration of RFD in rehabilitation models is necessary to begin to optimise neuromuscular function prior to return to sport. The next section will discuss the determinants of RFD and responses of RFD to training to aid in best practice.

\section{Evidence for determinants of RFD}

Essentially, RFD can be considered a composite function of a MIVF capabilities multiplied by the ability to express this available force generating capacity (i.e., relative RFD) at specific time points. Therefore, if there was no variability in relative RFD, then explosive strength would simply be scaled to MIVF and be influenced by the factors which explain this form of strength. 
The variability in relative RFD and as such the association to MIVF vary throughout the force-time curve with much higher variability in RFD during the early phase of contraction. Early phase RFD (0-50 ms) has been shown to vary by 8 -fold ${ }^{30}$. As such it's no surprise that poor relationships with RFD over 50 ms and MIVF have been documented, with explained variance of only $20-25 \%^{30,31}$. The relationship between MIVF and RFD increases with increasing time from onset with MIVF explaining 50 and $80 \%$ of the variability in RFD over $0-100$ and 0 $150 / 200$ ms respectively.

Other factors thought to explain RFD in the early phase of contraction include the level of agonist activation and intrinsic contractile properties 8,30 . The level of agonist activation has been shown to be sub-optimal in all individuals, and highly variable. Typically, most individuals on average can achieve $40 \%$ activation over the initial $50 \mathrm{~ms}$ from rest (assessed using surface Electromyography and comparison of electrically evoked to voluntary contractions) which ranges between $10-80 \%{ }^{30}$. Twitch contractile properties are thought to explain around 10$15 \%$ of the early phase variability in RFD, once its association with MIVF is accounted for ${ }^{30,31}$. Collectively, the early phase of force development is thought of as the ability to increase neuromuscular activation rapidly (explained variability, 50\%), the contractile responses to low levels of activation (twitch response, 15\%) and maximal force generating capacity (20-25\%). Mid-phase RFD is explained largely by MIVF (50\%) but with stronger associations with contractile responses to high pulse frequency electrical stimulation (evoked contractions of 8 pulse at $300 \mathrm{~Hz}$ which evokes the muscle tendon units peak evoked RFD), and limited associations with neuromuscular activation $(10-15 \%)^{30}$. This is thought to be due to fact that activation levels are already high/maximal (generally in excess of EMG amplitude at MIVF), and that the motor unit frequency required to achieve peak RFD is significantly higher than for MIVF (300 vs 30-50 Hz) ${ }^{8}$.

Other factors are considered to play a role in RFD, for example, both muscle architecture and fibre type composition are thought to contribute to RFD, but has received limited research attention. Despite initially considered to contribute to RFD ${ }^{27}$, muscle-tendon unit stiffness has been shown to report a spurious relationship with RFD though its association with MIVF ${ }^{32}$. There is an important consideration of contraction type influence on observed RFD. Tillin et al. ${ }^{33}$ reported RFD across isometric, concentric and eccentric contraction of the knee extensors on an acceleration controlled isokinetic machine. Concentric RFD was $60 \%$ higher than isometric or eccentric RFD after $25 \mathrm{~ms}$. Furthermore, participants achieved concentrically $77 \%$ of evoked force capacity after $50 \mathrm{~ms}$ compared to $36-46 \%$ isometrically and only $23 \%$ eccentrically. The superior ability to utilise the available force generating capacity during concentric contraction demonstrates an enhanced capability for agonist activation during rapid concen- tric tasks, which was further supported via elevated EMG amplitude during concentric versus isometric or eccentric contractions.

\section{Responses to training}

Resistance training utilising moderate to heavy loads (sustained contractions greater than $2 \mathrm{~s}$ ) has proved successful at developing MIVF, but there is equivocal evidence concerning its effects on RFD ${ }^{9,34}$. Resistance training studies using either slow increases in force and sustained contractions ${ }^{9}$ or isoinertial resistance training with moderate loads $(70 \%$ of one repetition maximum, 8-12 repetitions) have reported differential adaptations on the force-time curve with increased late phase RFD (>200 ms) and no change in RFD during the early phase $(<100 \mathrm{~ms})^{34}$. Tillin et al. ${ }^{6}$ suggested the enhanced agonist activation which explained enhanced MIVF after 4 weeks, was specific to the high force component of the force-time curve. A recent study compared the adaptations in RFD following high intensity resistance training (3 sets at 3-5 repetition maximum with 3 minutes' recovery between sets) against that of high volume resistance (3 sets, 10-12 repetitions at $70 \% 1$ repetition maximum), which can be considered moderate intensity resistance training. The Authors reported that the high intensity group elicited large increases in RFD during the early periods of force development after training (78\% increase in RFD over $50 \mathrm{~ms}$ and trend for increased RFD over $90 \mathrm{~ms},+59 \%, \mathrm{P}=0.052)^{35}$ whereas there was no change $(-3-4 \%)$ in RFD for the moderate intensity resistance training group. The Authors speculated that high intensity training elicited adaptation in the high threshold motor units, which resulted in increased RFD. Aagaard et al. ${ }^{9}$ reported enhanced RFD and agonist activation after 12 weeks of periodised resistance training in which the last four weeks involved heavy loads (4-6 repetition maximum). The differential effects versus a similar resistance programme of moderate loads ${ }^{34}$, which failed to elicit adaptations in RFD supports evidence to suggest that inclusion of a heavy load lifting ( $\leq 5$ repetition maximum) as part of a periodised resistance programme may be superior for enhancing the whole of the force-time curve and provide the necessary stimulus to enhance early phase agonist activation.

It is thought that intention to lift the weight or produce maximal force as quickly as possible is needed to enhance RFD ${ }^{36}$. Indeed, those studies which have used rapid maximal contractions from low force levels have shown enhanced agonist RFD accompanied by increased agonist activation (assessed using surface electromyography) ${ }^{28,29}$. Short term training involving rapid increase in force from rest has been shown to enhance RFD by $40-50 \%$ after only 4 weeks of training, with marked increased agonist activation (+40$50 \%)^{28,29}$.

The principle of intention to produce force as quickly as possible, may not appear to hold true with isoinertial resistance training, as Buckthorpe et al. ${ }^{37}$ report- 
The time has come to incorporate a greater focus on rate of force development training in the sports injury rehabilitation process

ed no change in RFD after three weeks of isoinertial resistance training with maximal intention to lift the weight during the concentric phase. The Authors suggested that rapid transition in force and activation might be the stimulus required to elicit changes in activation and RFD rather than merely intention. Isoinertial resistance training involves moderate levels of activation throughout the concentric and eccentric phases and does not involve increases in force from low to high levels and so in line with the principle of training specifity, so it reads training specifity phenomenon, may not train early phase RFD.

Maximal intention to lift lighter weights referred to as ballistic training has been shown to be more effective at enhancing RFD and is typically incorporated into programmes to train power and explosive strength. For example, Van Cutsem, et al. ${ }^{38}$ demonstrated a large increase in both RFD and motor unit discharge properties following 12 weeks of ballistic training at $30 \%$ one repetition maximum. Training resulted in no change in the recruitment sequence of motor units during ramp contractions (i.e., adhered before and after training to the size principle) ${ }^{39}$; an earlier onset of electro-myographic activity; increased maximal motor unit firing frequency and an increased incidence of motor unit doublets (inter-spike frequency $2-5 \mathrm{~Hz}$ ) in the electro-myographic signal during ballistic contractions. It appears that these may be the underlying mechanisms responsible for enhanced electro-myographic activity observed following explosive and ballistic training reported above.

As well as explosive strength training, sensorimotor training (which involves rapid low level contractions to re-stabilise joint complexes) has been shown to evoke marked increases in RFD (33\%) and agonist activation during the early phase of contraction (30$50 \mathrm{~ms}$ ) within the knee extensors, with no change in either MIVF or RFD measured at time periods greater than $100 \mathrm{~ms}$ (measured during isometric leg press) ${ }^{18}$, However, the benefits of this type of training are questionable when combined with resistance training and appear to be more related to training the ability to process incoming afferent information from joints, rather than efferent processing for joint stability.

\section{Summary and implications for rehabilitation and return to sport}

From the above, RFD appears more relevant than MIVF for short explosive contractions. Given that joint injury prevention likely is more reliant on rapid low level muscular contractions to timely re-stabilise joints and prevent or limit mechanical disruption, this may have important clinical relevance for injury and re-injury prevention programmes, whereas some sporting movements such as vertical jumping, with contraction times around $250 \mathrm{~ms}$ are likely influenced by late phase RFD and thus, more so the maximal strength capacity of the muscle. However, rehabilitation and screening prior to return to sport involves little in the way of testing and training RFD. From the small research, available it appears conventional rehabilitation fails to adequately restore RFD prior to return to sport and a greater focus on training and testing RFD may be needed to enhance return to sport outcomes and prevent re-injury risk.

For most, the ability to assess RFD during the rehabilitation period is problematic due to the required equipment (specifically designed isometric and/or isokinetic testing rigs) and testing skill. Assessment of RFD following injury such as anterior cruciate ligament rupture should involve single joint controlled assessment, with close consideration of the factors which can influence measurement. Limiting measurement error through a stiff measurement system and careful controlled assessment and interpretation of force-signals is required.

If RFD assessment is not possible, then appreciation of the determinants of RFD and responses to training should aid in enhancing patient outcomes following rehabilitation. Overall, it is hoped that this information will inform rehabilitation practices with a greater focus on RFD in the rehabilitation model. It is well recognised that rehabilitation should follow a phased approach based on criterion progression across five phases ${ }^{40}$. The early phases (one and two) should resolve pain and inflammation and restore range of motion, followed by a period of strength and muscular (and cardiovascular) endurance restoration (phase three). End stages (phases four) involve restoration of motor control and coordination, prior to on-field sports restoration, reconditioning and return to sport (phase five $)^{41}$. In this context, RFD training should be largely a focus of phase four, restoring neural activation and explosive strength prior to beginning sportspecific on-field rehabilitation. As part of a periodised strength training model, following a period of strength training to restore muscle physiological cross sectional area and maximal muscle strength, further training to enhance RFD to develop the functional capability to express muscle strength capacity explosively during fast sporting movements such as sprint running and rapid re-stabilisation of joint complexes following mechanical perturbation is required.

On the whole, it is recommended that return to sport neuromuscular re-training involve components to develop both maximal and explosive strength. Based on the determinants of strength, this should include a focus on development of maximal strength, as well as the explosive contractile responses of the muscle and the ability to rapidly increase neuromuscular activation. Conventional strength training with moderate load is effective at restoring maximal strength and a periodised resistance program with the final phased focused on heavy load lifting $(<5 R M)$ and eccentric strength would train both components of strength. Incorporation of partial squat heavy load lifting alongside full range of motion squat training was shown to result in a larger left and upward shift in the forcetime curve, resulting in increased impulse production during the early period of force development compared to full range of motion squat training only ${ }^{42}$ and could be a strategy to target both components of neu- 


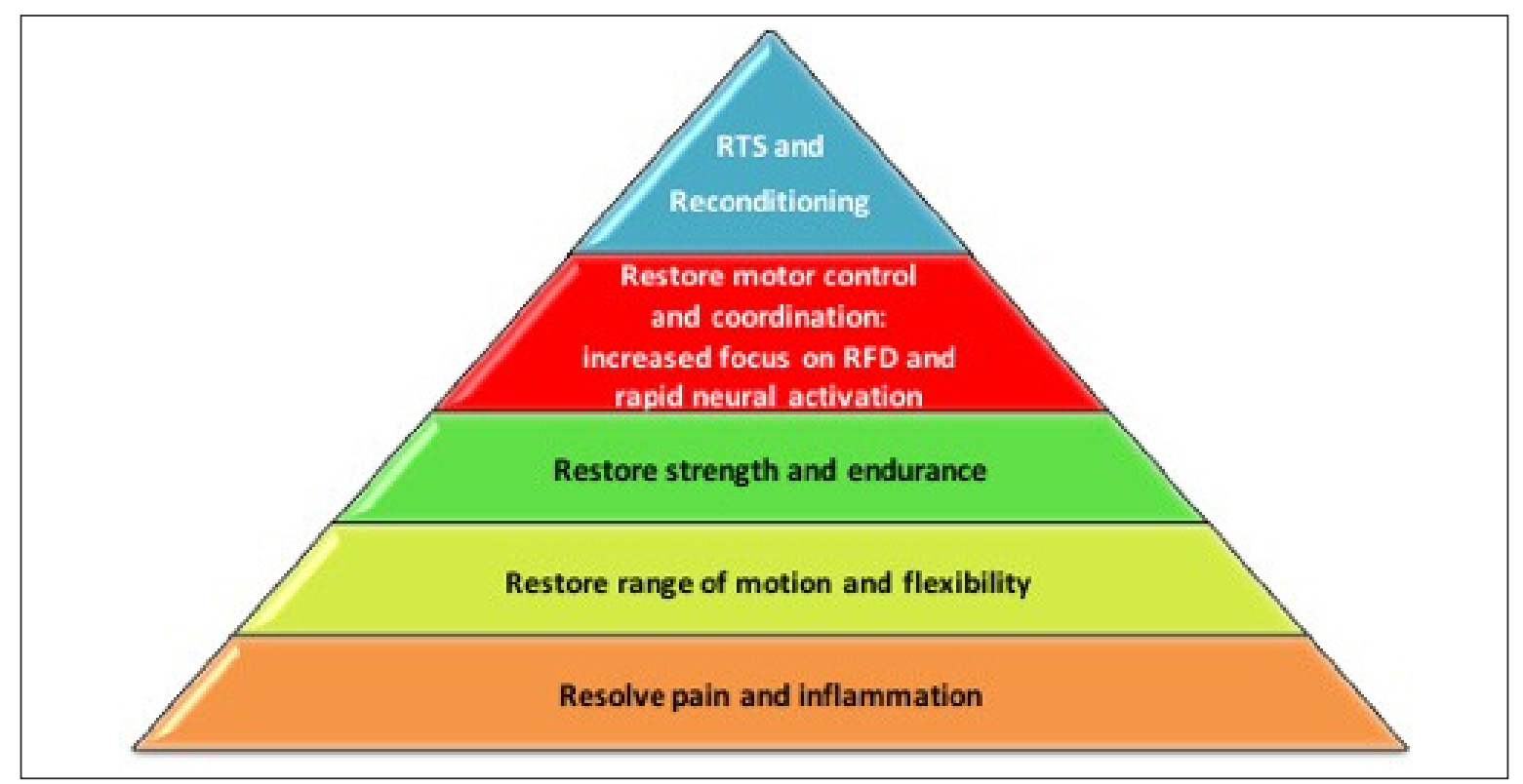

Figure 1. Rehabilitation model ${ }^{43}$ with increased focus in phase 4 on Rate of Force Development (RFD) through enhancing neural adaptations. ROM: Range of Motion; RTS: Return to Sport.

romuscular function. Heavy load lifting to achieve high levels of activation, and specifically to target the largest (and fastest) motor units appears necessary for maximal strength training to elicit adaptations in RFD. As such incorporation of heavy/maximal/supramaximal strength training alongside moderate resistance training may complement the neuromuscular adaptations seen from resistance training during the rehabilitation program. Further, incorporating explosive strength training to enhance the ability to develop force rapidly from low levels is required, whilst ballistic and plyometric training can further complement the program.

Obviously such a high load/ explosive strength and power training after injury must be proposed only after completion of the healing process, recovery of range of motion and flexibility, and applying the principle of progressive overload ${ }^{43}$. In clinical practice, we follow a periodised neuromuscular re-training programme with phase 4 split into 3 elements involving functional strength training and eccentric maximal strength, with a view to enhancing the ability to express isolated strength functionally and involving heavy load lifting and eccentric strength training; 4.2 explosive strength training and deceleration/landing control with a view to optimising neuromuscular preactivation and rapid neuromuscular activation at force onset and 4.3 involving plyometric training to develop the stretch shortening cycle and eccentric RFD/power. This is undertaken prior the commencement of onfield rehabilitation and return to sport to ensure sufficient explosive neuromuscular performance prior to commencement of high speed sporting actions (phase 5) (Fig. 1).

RFD training can be introduced earlier in the model than phase four with use of pool based rehabilitation, including jump and landing techniques in the swimming pool at various depths of water in line with a patient's functional strength recovery. With maximal intention to jump rapidly, there is a high transition in force from low to higher levels (as such it is expected there would also be a rapid transition in activation levels from low to high levels) which likely serve as an effective stimulus to develop RFD earlier in the rehabilitation programme (i.e., phase three, alongside a strengthening programme in the gym) with minimal ground reaction force. Ultimately optimally restoring neuromuscular function is a key goal in sports rehabilitation and incorporating a greater focus on RFD is one ingredient for this approach. Thus, we encourage clinicians to enhance their understanding of the topic and expertise at incorporating this aspect of training into their rehabilitation protocols/programmes.

\section{References}

1. Siff M. Biomechanical foundations of strength and power training. In: Zatsiorsky V ed Biomechanics in sports. London; Blackwell Sci Ltd. 2001:103-139.

2. Bisciotti GN, Quaglia A, Belli A, Carmimati G, Volpi P. Return to sports after ACL reconstruction: a new functional test protocol. Muscles Ligaments Tendons J. 2016;6(4):499-509.

3. Dauty M, Menu P, Fouasson-Chailloux A, Ferreol S, Dubois C. Prediction of hamstring injury in professional soccer players by isokinetic measures. Muscles Ligaments Tendons J. 2016;6 (1):116-123.

4. Beneke R, Taylor MJ. What gives Bolt the edge-A.V. Hill knew it already! J Biomech. 2010;43(11):2241-2243.

5. Krosshaug T, Nakamae A, Boden BP, et al. Mechanisms of anterior cruciate ligament injury in basketball: video analysis of 39 cases. Am J Sports Med. 2007;35:359-367.

6. Tillin NA, Pain MTG, Folland JP. Short-term unilateral resistance training affects the agonist-antagonist but not the force- 
The time has come to incorporate a greater focus on rate of force development training in the sports injury rehabilitation process

agonist activation relationship. Muscle Nerve. 2011;43:375384.

7. Padulo J, Oliva F, Frizziero A, Maffulli N. Muscles, Ligaments and Tendons Journal - Basic principles and recommendations in clinical and field science research: 2016 update. MLTJ. 2016;6(1):1-5.

8. Maffiuletti NA, Aagaard P, Blazevich AJ, Folland J, Tillin N, Duchateau J. Rate of force development: physiological and methodological considerations. Eur J Appl Physiol. 2016; 116(6):1091-1116.

9. Aagaard P, Simonsen EB, Andersen JL, Magnusson P, Dyhre-Poulsen $P$. Increased rate of force development and neural drive of human skeletal muscle following resistance training. J Appl Physiol. 2002;93:1318-1326.

10. Tillin NA, Jimenez-Reyes P, Pain MT, Folland JP. Neuromuscular performance of explosive power athletes versus untrained individuals. Med Sci Sports Exerc. 2010;42:781-790.

11. Tillin NA, Pain MTG, Folland JP. Explosive force production during isometric squats correlates with athletic performance in rugby union players. J Sports Sci. 2013;31:66-76.

12. Padulo J, Oliva F, Frizziero A, Maffulli N. Muscles, Ligaments and Tendons Journal. Basic principles and recommendations in clinical and field science research. MLTJ. 2014;3(4):250252.

13. West DJ, Owen NJ, Jones MR, et al. Relationships between force-time characteristics of the isometric midthigh pull and dynamic performance in professional rugby league players. J Strength Cond Res. 2011;25(11):3070-3075.

14. De Ruiter CJ, Leeuwen DV, Heijblom A, Bobbert MF, de Haan A. Fast isometric knee extension torque development and bilateral jump height. Med Sci Sports Exerc. 2006;38:18431853.

15. Nuzzo JL, McBride JM, Cormie P, McCaulley GO. Relationship between countermovement jump performance and multijoint isometric and dynamic tests of strength. J Strength Cond Res. 2008;22:699-707.

16. Solomonow M, Krogsgaard M. Sensorimotor control of knee stability. A review. Scand J Med Sci Sports. 2001;11(2):64-80.

17. Izquierdo M, Aguado X, Gonzalez R, Lopez JL, Hakkinen K. Maximal and explosive force production capacity and balance performance in men of different ages. Eur J Appl Physiol Occup Physiol. 1999;79:260-267.

18. Gruber M, Gollhofer A. Impact of sensorimotor on the rate of force development and neural activation. Eur J Appl Physiol. 2004;92:98-105.

19. Hubscher M, Zech A, Pfeifer K, Hänsel F, Vogt L, Banzer W. Neuromuscular training for sports injury prevention: a systematic review. Med Sci Sports Exerc. 2010;42:412-421.

20. Hawkins RD, Hulse MA, Wilkinson C, Hodson A, Gibson M. The association football medical research programme: an audit of injuries in professional football. Br J Sports Med. 2001;35: 43-47.

21. Buckthorpe MW, Pain MTG \& Folland JP. Central fatigue contributes to the greater reductions in explosive than maximum strength with high intensity fatigue. Exp Physiol. 2014;99:964973.

22. Angelozzi M, Madama M, Corsica C, et al. Rate of force development as an adjunctive outcome measure for return-to-sport decisions after anterior cruciate ligament reconstruction. J Orthop Sports Phys Ther. 2012;42(9):772-780.

23. Opar DA, Williams MD, Timmins RG, Dear NM, Shield AJ. Rate of torque and electromyographic development during anticipated eccentric contraction is lower in previously strained hamstrings. Am J Sports Med. 2013;41:116-125.

24. Ardern CL, Webster KE, Taylor NF, Feller JA. Return to the preinjury level of competitive sport after anterior cruciate ligament reconstruction surgery two-thirds of patients have not re- turned by 12 months after surgery. Am J Sports Med. 2011;39 (3):538-543.

25. Opar DA, Williams MD, Shield AJ. Hamstring strain injuries: factors that lead to injury and re-injury. Sports Med. 2012;42(3):209-226.

26. Buckthorpe MW, Hannah R, Pain MTG, Folland JP. Reliability of Neuromuscular Measurements during Explosive Isometric Contractions, with Special Reference to EMG Normalisation Techniques. Muscle Nerve. 2012;46:566-576.

27. Bosjen-Moller J, Magnusson SP, Rasmussen LR, Kjaer M, Aagaard P. Muscle performance during maximal isometric and dynamic contractions is influenced by the stiffness of the tendinous structures. J Appl Physiol. 2005;99:986-994.

28. Tillin NA, Pain MTG, Folland JP. Short-term training for explosive strength causes neural and mechanical adaptations. Exp Physiol. 2012;97:630-641.

29. Del Balso C, Cafarelli E. Adaptations in the activation of human skeletal muscle induced by short-term isometric resistance training. J Appl Physiol. 2007;103:402-411.

30. Folland JP, Buckthorpe MW, Hannah R. Human capacity for explosive force production: Neural and contractile determinants. Scand J Med Sci in Sports. 2014;24:894-906.

31. Andersen LL, Aagaard P. Influence of maximal muscle strength and intrinsic muscle contractile properties on contractile rate of force development. Eur J Appl Physiol. 2006;96:4652.

32. Hannah R, Folland JP. Muscle-tendon unit stiffness does not independently affect voluntary explosive force production or muscle intrinsic contractile properties. Appl Physiol Nutr Metab. 2015:40:87-95.

33. Tillin NA, Pain MTG, Folland JP. Contraction type influences the human ability to use the available torque capacity of skeletal muscle during explosive efforts. Proc Biol Sci. 2012;7:228 (1736):2106-2115.

34. Andersen LL, Andersen JL, Kebis MK, Aagaard P. Early and late rate of force development: differential adaptive responses to resistance training? Scand J Med Sci Sports. 2010;20 (1):e162-e169.

35. Mangine GT, Hoffman JR, Wang R, et al. Resistance training intensity and volume affect changes in rate of force development in resistance trained men. Eur J Appl Physiol. 2016; 116:2367-2374.

36. Behm DG, Sale DG. Intended rather than actual movement velocity determines velocity-specific training response. J Appl Physiol. 1993;74:359-368.

37. Buckthorpe MW, Erskine R, Fletcher G \& Folland JP. Neural adaptations explain the task specificity of strength changes after resistance training. Scand J Med Sci Sports. 2015;25 (5):640-649.

38. Van Cutsem M, Duchateau J, Hainaut K. Changes in single motor unit behaviour contribute to the increase in contraction speed after dynamic training in humans. J Physiol. 1998; 513 (Part 1): 295-305.

39. Henneman E. Relation between size of neurons and their susceptibility to discharge. Science. 1957;126;1345-1347.

40. Zanobbi M, Fazzini D, Marcheggiani GM. Rehabilitation in five phases. In: Roi GS, Della Villa S eds. Football medicine strategies for knee injuries. Calzetti Mariucci Editore, Torgiano, 2012, pp. 53-54.

41. Della Villa S, Boldrini L, Ricci M, et al. Clinical Outcomes and Return-to-Sports Participation of 50 Soccer Players After Anterior Cruciate Ligament Reconstruction Through a SportSpecific Rehabilitation Protocol. Sports Health. 2012;4:17-24.

42. Bazyler CD, Sato K, Wassinger CA, Lamont HS, Stone MH The efficacy of incorporating partial squats in maximal strength training. J Strength Cond Res. 2014; 28:3024-3032.

43. Houglum PA. Therapeutic exercise for musculoskeletal injuries. Human Kinetics, Champaign, IL, 2010. 